\title{
Problème de Lehmer pour les hypersurfaces de variétés abéliennes de type C.M.
}

\author{
par \\ Nicolas RATAZZi (Paris)
}

1. Introduction. On sait depuis les travaux de Philippon [10]-[12], puis Bost-Gillet-Soulé [3] dans le cadre de l'intersection arithmétique, comment définir la hauteur des variétés projectives, l'idée étant de considérer un point comme une variété de dimension zéro et de généraliser ceci en dimension supérieure. De même que dans le cas des points, on sait pour les variétés abéliennes munies d'un fibré en droites ample et symétrique définir une hauteur particulièrement agréable : la hauteur canonique $\widehat{h}_{\mathcal{L}}$, ou hauteur normalisée. En dimension zéro, il existe un théorème caractérisant les points de hauteur normalisée nulle; c'est un résultat de Kronecker dans le cas de $\mathbb{G}_{m}$.

Philippon [12] dans le cas d'un produit de courbes elliptiques, puis Zhang [20] et David-Philippon [5] dans le cas général ont montré comment généraliser ce résultat pour caractériser les sous-variétés de hauteur normalisée nulle : ce sont les translatées d'une sous-variété abélienne par un point de torsion. On dit qu'une telle sous-variété est une sous-variété de torsion. La réponse à cette question résoud une conjecture de Bogomolov, qui dans sa formulation initiale a été démontrée en premier par Ullmo [18].

Ceci étant, on peut se demander comment minorer la hauteur normalisée d'une sous-variété de hauteur non nulle d'une variété abélienne. Dans leur article [5], David et Philippon ont formulé un problème général (le problème 1.7) contenant cette question. En terme du degré défini ci-dessous, on peut notamment faire ressortir de la discussion suivant la formulation de leur problème l'énoncé suivant :

Conjecture 1 (David-Philippon). Soit A une variété abélienne définie sur un corps de nombres $k$, munie d'un fibré ample et symétrique $\mathcal{L}$. Soit $V$ une sous-variété stricte de $A$ sur $k, k$-irréductible et qui n'est pas réunion

2000 Mathematics Subject Classification: 11G50, 14G40, 14K12, $14 \mathrm{~K} 22$.

Key words and phrases: abelian varieties, normalised height, Lehmer problem. 
de sous-variétés de torsion. Alors, on a l'inégalité

$$
\frac{\widehat{h}_{\mathcal{L}}(V)}{\operatorname{deg}_{\mathcal{L}}(V)} \geq c(A / k, \mathcal{L}) \operatorname{deg}_{\mathcal{L}}(V)^{-1 /(s-\operatorname{dim} V)},
$$

où $s$ est la dimension du plus petit sous-groupe algébrique contenant $V$, et où $c(A / k, \mathcal{L})$ est une constante ne dépendant que de $A / k$ et de $\mathcal{L}$.

1.1. Degré et hauteur. Soient $k$ un corps de nombres supposé plongé dans $\mathbb{C}$, et $\mathcal{O}_{k}$ son anneau d'entiers. On dira que $V$ est une variété algébrique sur $k$ si $V$ est un $k$-schéma de type fini géométriquement réduit. On dira que $G$ est un groupe algébrique sur $k$ si c'est une variété en groupes sur $k$. On dira que $A$ est une variété abélienne définie sur $k$ si c'est un groupe algébrique connexe propre et lisse sur $k$. Par sous-variété on entendra toujours sousvariété fermée.

DÉfinition 1. On dit qu'une variété abélienne simple $A / k$ sur un corps de nombres est de type C.M. si son anneau d'endomorphismes tensorisé par $\mathbb{Q}$ contient (après éventuellement extension du corps de base) un corps commutatif $F$ de dimension $2 \operatorname{dim} A$ sur $k$. Une variété abélienne $A / k$ est dite de type C.M. si son anneau d'endomorphismes tensorisé par $\mathbb{Q}$ contient un produit de corps de nombres $K_{1} \times \cdots \times K_{r}$ tels que $\sum\left[K_{i}: \mathbb{Q}\right]=2 \operatorname{dim} A$.

Soit $X$ une variété projective munie d'un plongement $\varphi_{\mathcal{L}}: X \hookrightarrow \mathbb{P}_{k}^{n}$ défini par un fibré $\mathcal{L}$ très ample sur $X$. Si $\mathcal{O}(1)$ dénote le fibré standard sur $\mathbb{P}_{\mathcal{O}_{k}}^{n}$, on a $\varphi_{\mathcal{L}}^{*} \mathcal{O}(1)_{k} \simeq \mathcal{L}$. On note $\overline{\mathcal{O}(1)}$ le fibré standard muni de la métrique de Fubini-Study. Si $V$ est une sous-variété de $X$, on note $\mathcal{V}_{\mathcal{L}}$ l'adhérence schématique de $\varphi_{\mathcal{L}}(V)$ dans $\mathbb{P}_{\mathcal{O}_{k}}^{n}$.

DÉfinition 2. Si $\mathcal{L}$ est un fibré ample sur une variété abélienne $A$, et $V$ une sous-variété de $A$, on appelle le degré de la variété $V$ relativement à $\mathcal{L}$, et on note $\operatorname{deg}_{\mathcal{L}}(V)$, l'entier $\operatorname{deg}\left(c_{1}(\mathcal{L})^{\operatorname{dim} V} \cdot V\right)$ où deg est le degré projectif usuel d'un 0-cycle.

DÉfinition 3. On appelle hauteur de la variété $V$ associée à $\mathcal{L}$, et on note $h_{\mathcal{L}}(V)$, le réel $h_{\overline{\mathcal{O}(1)}}\left(\mathcal{V}_{\mathcal{L}}\right)$ où $h_{\overline{\mathcal{O}(1)}}(\cdot)$ est la hauteur, au sens de BostGillet-Soulé [3], associée au fibré hermitien $\overline{\mathcal{O}(1)}$.

Remarque 1. Par le théorème 3 , p. 366 , de [17], $h_{\mathcal{L}}(V)$ coïncide avec la hauteur $h\left(f_{V, \mathcal{L}}\right)$ de Philippon, telle que définie au paragraphe 2 de [12], où $f_{V, \mathcal{L}}$ est une forme éliminante de l'idéal de définition de $\varphi_{\mathcal{L}}(V)$ dans $k\left[X_{0}, \ldots, X_{n}\right]$. (Le terme d'erreur de [17] disparaît du fait du changement de normalisation pour la hauteur de Philippon entre les articles [10] et [12].)

DÉfinition 4. Dans le cas où $X=A$ est une variété abélienne, et où $\mathcal{L}$ est de plus symétrique, Philippon [12], puis Zhang [19] avec des méthodes arakeloviennes, ont montré en utilisant un procédé de limite à la NéronTate, comment définir une hauteur canonique, notée $\widehat{h}_{\mathcal{L}}(\cdot)$, sur l'ensemble 
des sous-variétés de $A$. Cette hauteur vérifie notamment : si $X$ est une sous-variété de $A$, de stabilisateur $G_{X}$, et si $n$ est un entier, alors

$$
\widehat{h}_{\mathcal{L}}([n](X))=\frac{n^{2(\operatorname{dim} X+1)}}{\left|\operatorname{ker}[n] \cap G_{X}\right|} \widehat{h}_{\mathcal{L}}(X) .
$$

DÉfinition 5. Soit $A / k$ une variété abélienne. On dit que $V$ est une sous-variété de torsion de $A$ si $V=a+B$ avec $a \in A_{\text {tors }}$ et $B$ une sousvariété abélienne de $A$.

D'après les résultats de Philippon [12], David-Philippon [5] et Zhang [19], on a, si $V$ est une sous-variété de $A / k$ définie sur une extension finie $K / k$,

$$
\widehat{h}_{\mathcal{L}}(V)=0 \quad \text { si et seulement si } \quad V \text { est une sous-variété de torsion. }
$$

DÉfinition 6. Soient $V$ une sous-variété de $A$ sur $k$, et $\theta$ un nombre réel positif. On pose $V(\theta, \mathcal{L})=\left\{x \in V(\bar{k}) \mid \widehat{h}_{\mathcal{L}}(x) \leq \theta\right\}$. On appelle alors le minimum essentiel de $V$, et on note $\widehat{\mu}_{\mathcal{L}}^{\text {ess }}(V)$, le réel

$$
\widehat{\mu}_{\mathcal{L}}^{\text {ess }}(V)=\inf \{\theta>0 \mid \overline{V(\theta, \mathcal{L})}=V\} .
$$

1.2. Résultats. Dans la direction de la conjecture 1 , on a le résultat suivant (cf. corollaire 2 de [14]) :

ThÉORÈme 1. Si A est une variété abélienne de type C.M., $\mathcal{L}$ un fibré en droites ample et symétrique de $A$, et si $V$ est une sous-variété algébrique stricte de $A$ sur $k, k$-irréductible et qui n'est pas réunion de sous-variétés de torsion, alors on a l'inégalité

$$
\frac{\widehat{h}_{\mathcal{L}}(V)}{\operatorname{deg}_{\mathcal{L}}(V)} \geq \widehat{\mu}_{\mathcal{L}}^{\operatorname{ess}}(V) \geq c(A / k, \mathcal{L}) \operatorname{deg}_{\mathcal{L}}(V)^{-1 /(n-\operatorname{dim} V)}\left(\log \left(3 \operatorname{deg}_{\mathcal{L}}(V)\right)\right)^{-\kappa(n)}
$$

où $n$ est la dimension du plus petit sous-groupe algébrique contenant $V$, et où $\kappa(n)$ est une constante effectivement calculable ne dépendant que de $n$ (par exemple $\kappa(n)=(2 n(n+1) !)^{n+2}$ convient $)$.

On se restreint dans cet article au cas particulier des hypersurfaces $V$ d'une variété abélienne de type C.M. Dans ce cas et sous les hypothèses du théorème précédent, on a nécessairement $n=g$. En effet, par définition $n$ appartient à $\{g-1, g\}$. De plus, si $n$ était égal à $g-1$, alors $V$ serait une réunion de sous-variétés de torsion, ce qui contredit l'hypothèse faite sur $V$. Ainsi, dans le cas des hypersurfaces, la conjecture est la suivante :

Conjecture 2. Sous les hypothèses précédentes, et en supposant de plus que $V$ est hypersurface de $A$, on a l'inégalité

$$
\widehat{h}_{\mathcal{L}}(V) \geq c(A / k, \mathcal{L})
$$

où $c(A / k, \mathcal{L})$ est une constante ne dépendant que de $A / k$ et de $\mathcal{L}$.

De même, le théorème 1 se spécialise en 
THÉORÈme 2. Sous les hypothèses précédentes, et en supposant que $V$ est une hypersurface de $A$, on a l'inégalité

$$
\widehat{h}_{\mathcal{L}}(V) \geq \operatorname{deg}_{\mathcal{L}}(V) \widehat{\mu}_{\mathcal{L}}^{\text {ess }}(V) \geq c(A / k, \mathcal{L})\left(\log \left(3 \operatorname{deg}_{\mathcal{L}}(V)\right)\right)^{-\kappa(g)},
$$

où $g$ est la dimension de $A$, et où $\kappa(g)$ est une constante effectivement calculable ne dépendant que de $g$ (par exemple $\kappa(g)=(2 g(g+1) !)^{g+2}$ convient).

Dans ce cadre restreint aux hypersurfaces, on montre un résultat sensiblement plus fin en direction de la conjecture 2 : on peut prendre pour $\kappa$ une valeur absolue, indépendante de $g$. En notant $\delta_{i, j}$ le symbole de Kronecker (valant 1 si $i=j$ et 0 sinon), on démontre ici le résultat suivant :

ThÉORÈme 3. Si A est une variété abélienne de type C.M., $\mathcal{L}$ un fibré en droites ample et symétrique de $A$, et si $V$ est une hypersurface irréductible de $A$ sur $k$ qui n'est pas réunion de sous-variétés de torsion, alors on a l'inégalité

$$
\widehat{h}_{\mathcal{L}}(V) \geq \operatorname{deg}_{\mathcal{L}}(V) \widehat{\mu}_{\mathcal{L}}^{\mathrm{ess}}(V) \geq c(A / k, \mathcal{L}) \frac{\left(\log \log \operatorname{deg}_{\mathcal{L}}(V)\right)^{1+2 \delta_{g-s, 1}}}{\left(\log \operatorname{deg}_{\mathcal{L}}(V)\right)^{2+\delta_{g-s, 1}}},
$$

où $s$ est la dimension du stabilisateur de $V$.

Notons que $\delta_{g-s, 1}=0$ sauf si $A / k$ est le produit $E \times B$ d'une courbe elliptique $E / k$ et d'une variété abélienne $B / k$, et si $V$ est de la forme $\overline{\{P\}} \times B$, où $P$ est un point $\bar{k}$-rationnel de $E$ qui n'est pas de torsion. Dans ce cas, en supposant que $A / k$ est une courbe elliptique, $\mathcal{L}$ le fibré associé au diviseur $3(0)$, et où $V=\overline{\{P\}}$ est l'ensemble des conjugués d'un point non de torsion $P \in A(K)$ dans une extension finie $D=[K: k]$, on retrouve exactement le résultat de Laurent [9] sur le problème de Lehmer elliptique, à savoir

$$
\widehat{h}(P) \geq \frac{c(A)}{D}\left(\frac{\log \log D}{\log D}\right)^{3}
$$

Dans le cas d'une "vraie" hypersurface (i.e., quand $\delta_{g-s, 1}=0$ ), on obtient une minoration un peu meilleure.

La démonstration suit fondamentalement les idées (et reprend une grande partie des preuves) de l'article de David-Hindry [4] concernant le problème de Lehmer pour les points d'une variété abélienne. Il s'agit en fait d'une extension d'un travail de Amoroso-David [1] concernant le cas des tores, au cas des variétés abéliennes de type C.M. On fait un raisonnement par l'absurde, et on se fixe une hypersurface $V$ contredisant la conclusion du théorème. La preuve consiste essentiellement en une preuve de transcendance classique.

On commence tout d'abord par construire une fonction auxiliaire, nulle avec un grand ordre sur $V$. Pour cela on met en oeuvre une astuce dûe à Amoroso-David (qu'ils introduisent dans [1]) permettant de se ramener à un système d'équations fini et de hauteur controlée. Ceci nous permet d'appliquer un lemme de Siegel pour construire la fonction auxiliaire $F$. 
La deuxième partie de la preuve, l'extrapolation, consiste à montrer que $F$ continue à s'annuler avec un ordre relativement grand sur les transformées $\alpha_{v}(V)$ de $V$ par certaines isogénies $\alpha_{v}$ où $v$ décrit un ensemble de places finies convenables du corps de définition $k$ (les $\alpha_{v}$ sont des relevées sur $A / k$ des morphismes de Frobenius en caractéristique finie $p_{v}$; c'est pour assurer l'existence de ces isogénies que l'on se restreint au cas C.M.). Il s'agit d'une extrapolation aux places $v$-adiques. L'idée pour montrer ceci est d'appliquer une généralisation du petit théorème de Fermat : c'est la méthode employée pour la première fois par Dobrowolski [6] dans le cas du problème de Lehmer sur $\mathbb{G}_{m}$. Cette idée a ensuite été reprise par Laurent [9] dans le cas des courbes elliptiques à multiplication complexes puis étendue au cas des variétés abéliennes de type C.M. par David-Hindry [4]. C'est cette dernière généralisation que nous allons reprendre.

Ceci étant fait, il suffit pour conclure d'appliquer le théorème de Bézout géométrique pour aboutir à une contradiction (pour peu que les différents paramètres intervenant dans l'étape de transcendance aient étés convenablement choisis). Pour cette dernière étape, on a besoin d'avoir une bonne minoration du degré de l'union des $\alpha_{v}(V)$. Ceci se fait en suivant les calculs de $[4]$.

Remerciements. Je tiens à remercier Sinnou David pour m'avoir suggéré l'écriture de cet article, et je tiens également à remercier Marc Hindry pour les nombreuses discussions que nous avons eues sur le sujet.

\section{Frobenius, isogénies admissibles et dérivations}

2.1. Morphismes de Frobenius. On commence par introduire quelques notations :

Si $k$ est un corps de nombres, on note $\mathcal{O}_{k}$ son anneau d'entiers, $v$ une place finie de $k$, et $k_{v}$ le corps résiduel associé à $v$.

Si $A / k$ est une variété abélienne, on note $\mathcal{A} / \mathcal{O}_{k}$ son modèle de Néron, et $A_{v} / k_{v}$ la fibre spéciale correspondant à la place finie $v$. Rappelons la propriété universelle du modèle de Néron : si $\mathcal{X} / \mathcal{O}_{k}$ est lisse, de fibre générique $X / k$, tout $k$-morphisme $X \rightarrow A$ se relève de manière unique en un $\mathcal{O}_{k^{-}}$ morphisme $\mathcal{X} \rightarrow \mathcal{A}$.

Sur la variété $A_{v} / k_{v}$, on dispose d'un endomorphisme particulier : le morphisme de Frobenius Frob $_{v}$, correspondant en coordonnées projectives à l'élévation à la puissance $q=\mathrm{N}(v)$, où $\mathrm{N}(v)$ est la norme $K / \mathbb{Q}$ de $v$.

La propriété universelle du produit fibré $A_{v}=\mathcal{A} \times \mathcal{O}_{k} k_{v}$ permet d'associer naturellement à tout $\mathcal{O}_{k}$-endomorphisme de $\mathcal{A}$ un $k_{v}$-endomorphisme de $A_{v}$. En utilisant la propriété universelle du modèle de Néron, on en déduit une flèche naturelle

$$
\Psi: \operatorname{End}_{k}(A) \rightarrow \operatorname{End}_{k_{v}}\left(A_{v}\right)
$$


Cette flèche n'est en général pas surjective, mais on peut par contre montrer qu'elle est injective aux places de bonne réduction. Dans le cas C.M., un théorème de Shimura-Taniyama permet d'affirmer que le morphisme Frob $v$ se relève en presque toutes places:

Proposition 1 (Shimura-Taniyama). Soit $A / k$ une variété abélienne de type C.M. Notons $\prod_{i=1}^{r} K_{i}$ le produit de corps de nombres qui est inclus dans $\operatorname{End}_{k}(A) \otimes \mathbb{Q}$ et tel que $\sum_{i=1}^{r}\left[K_{i}: \mathbb{Q}\right]=2 \operatorname{dim} A$. On suppose que le corps de nombres $k$ contient tous les $K_{i}$, et que $\prod_{i=1}^{r} \mathcal{O}_{K_{i}}$ est inclus dans $\operatorname{End}_{k}(A)$. Alors, pour presque toutes places, l'endomorphisme Frob $_{v}$ se relève en un $k$-endomorphisme $\alpha_{v}$ de $A$. On appelera morphisme de Frobenius sur $A$ un tel endomorphisme.

Démonstration. C'est le théorème 1, paragraphe III.13, de [16].

Ce sont ces morphismes de Frobenius sur $A / k$ qui vont nous permettre d'écrire l'étape d'extrapolation.

Remarque 2. En fait on pourrait spécifier les places qu'il faut exclure dans la proposition, mais nous n'en aurons pas besoin. Par ailleurs, pour pouvoir appliquer le théorème, il faut vérifier deux conditions : la première est toujours satisfaite quitte à faire une extension de degré borné de $k$. La seconde n'est pas toujours satisfaite, mais on peut toujours trouver une variété abélienne isogène qui la vérifie.

Quitte à faire une extension de degré borné de $k$, et quitte à prendre une variété abélienne isogène à la variété de départ, on supposera désormais toujours que les hypothèses de la proposition 1 sont satisfaites.

2.2. Isogénies admissibles. On rappelle la notion d'isogénie admissible telle qu'introduite dans [4].

DÉfinition 7. Soient $A$ une variété abélienne et $\mathcal{L}$ un fibré ample sur $A$. Une isogénie $\alpha$ de $A$ est dite admissible par rapport à $\mathcal{L}$ si

(1) $\alpha$ est dans le centre de $\operatorname{End}(A)$,

(2) il existe un entier $\mathrm{q}(\alpha)$, appelé poids de $\alpha$, tel que $\alpha^{\star} \mathcal{L} \simeq \mathcal{L}^{\otimes \mathrm{q}(\alpha)}$.

Remarque 3. En fait la condition (1) ne sert qu'à simplifier l'énoncé du lemme 3. C'est la condition (2) qui importe vraiment. Les seules isogénies qui nous intéresseront sont les relevées $\alpha_{v}$ des morphismes de Frobenius qui sont admissibles (cf. la proposition 2).

Lemme 1. Soient $A$ une variété abélienne de dimension g munie d'un fibré en droites très ample $\mathcal{L}$, et $\alpha$ une isogénie admissible relativement à $\mathcal{L}$, de poids $q=\mathrm{q}(\alpha)$. Dans le plongement projectif de $A$, associé à $\mathcal{L}, A \hookrightarrow \mathbb{P}_{n}$, on $a$ :

(1) $\operatorname{card}(\operatorname{ker}(\alpha))=q^{g}$, 
(2) pour toute sous-variété $V$ de $A$ de stabilisateur $G_{V}$, on a

$$
\operatorname{deg}_{\mathcal{L}}(\alpha(V))=\frac{q^{\operatorname{dim} V}}{\left|G_{V} \cap \operatorname{ker}(\alpha)\right|} \operatorname{deg}_{\mathcal{L}}(V) .
$$

Démonstration. Le point (1) est facile : par définition, $\alpha^{\star} \mathcal{L} \simeq \mathcal{L}^{\otimes q}$, d'où

$$
q^{g} \operatorname{deg}_{\mathcal{L}}(A)=\operatorname{deg}_{\mathcal{L} \otimes q}(A)=\operatorname{deg}_{\alpha^{\star} \mathcal{L}}(A)=|\operatorname{ker}(\alpha)| \operatorname{deg}_{\mathcal{L}}(A) .
$$

L'amplitude de $\mathcal{L}$ nous assure que le dernier degré est strictement positif. On simplifie pour conclure. Pour le point (2), il s'agit du lemme 6(ii) de [7].

Lemme 2. Soient $G$ un sous-groupe algébrique de la variété abélienne $A / k, \mathcal{L}$ un fibré très ample sur $A$, et $\alpha$ une isogénie admissible relativement $\grave{a} \mathcal{L}$ de poids $\mathrm{q}(\alpha)$ de $A$. On a

$$
\mathrm{q}(\alpha)^{\operatorname{dim} G} \leq \operatorname{card}(\operatorname{ker}(\alpha) \cap G) \leq\left[G: G^{0}\right] \mathrm{q}(\alpha)^{\operatorname{dim} G} .
$$

Démonstration. On note que

$$
\left[G: G^{0}\right] \operatorname{card}\left(\operatorname{ker}(\alpha) \cap G^{0}\right) \geq \operatorname{card}(\operatorname{ker}(\alpha) \cap G) \geq \operatorname{card}\left(\operatorname{ker}(\alpha) \cap G^{0}\right) .
$$

La restriction de $\alpha$ à la sous-variété abélienne $G^{0}$ est encore une isogénie admissible de poids $\mathrm{q}(\alpha)$ pour $\left(G^{0},\left.\mathcal{L}\right|_{G^{0}}\right)$ (cf. lemme 2.4(ii) de [4]). Par le lemme 1(1), on en déduit que le cardinal du noyau de cette isogénie $\left.\alpha\right|_{G^{0}}$ est $\mathrm{q}(\alpha)^{\operatorname{dim} G^{0}}$.

Soit $V$ une sous- $k$-variété stricte de $A, k$-irréductible. Le lemme suivant (dont l'origine remonte à Dobrowolski [6]) montre que les images par une isogénie admissible de ses composantes géométriquement irréductibles sont essentiellement distinctes. On commence pour cela par donner une définition :

DÉfinition 8. Soient $A$ une variété abélienne et $\mathcal{L}$ un fibré en droites ample sur $A$. Deux isogénies admissibles de $A$ par rapport à $\mathcal{L}$ sont dites premières entre elles si leurs poids sont premiers entre eux.

Lemme 3. Soient $A$ une variété abélienne sur $k$ de dimension $g \geq 1$, $\mathcal{L}$ un fibré en droites très ample sur $A, V$ une sous-k-variété stricte de $A$, irréductible sur $k$. Si $V$ n'est pas une réunion de sous-variétés de torsion de A, on a :

(1) Pour tout couple $(\alpha, \beta)$ d'isogénies admissibles pour $\mathcal{L}$, de poids distincts, pour tout $\sigma \in \operatorname{Gal}(\bar{k} / k)$, et pour toute composante géométriquement irréductible $W$ de $V$, les sous-variétés $\alpha(W)$ et $\beta(\sigma(W))$ sont distinctes.

(2) Soit $\mathcal{P}$ un ensemble d'isogénies admissibles pour $\mathcal{L}$, deux à deux premières entre elles. Notons $V_{1}, \ldots, V_{M}$ les composantes géométriquement irréductibles de $V$, et notons $\mathcal{Q}$ le sous-ensemble de $\mathcal{P}$ défini par

$$
\mathcal{Q}=\left\{\alpha \in \mathcal{P} \mid \exists i, j, 1 \leq i<j \leq M, \alpha\left(V_{i}\right)=\alpha\left(V_{j}\right)\right\} .
$$

Le cardinal de $\mathcal{Q}$ est majoré par $\log M / \log 2$. 
Démonstration. Dans ce contexte il s'agit de la proposition 2.7 de [4].

On conclut ce paragraphe en "rappelant" que les morphismes de Frobenius sur $A / k$ sont des isogénies admissibles :

DÉfinition 9. Soient $A$ une variété abélienne et $\mathcal{L}$ un fibré en droites ample sur $A$. Suivant Mumford, on dit que $\mathcal{L}$ est totalement symétrique si $\mathcal{L}$ est le carré d'un fibré symétrique.

Le théorème de Lefschetz (cf. par exemple le théorème A.5.3.6 de [8]) nous indique que si $\mathcal{L}$ est un fibré en droites ample, alors $\mathcal{L}^{\otimes 3}$ est très ample.

Proposition 2. Soient $A / k$ une variété abélienne de type C.M. vérifiant les hypothèses de la proposition 1 , et $\mathcal{L}$ un fibré en droites très ample et totalement symétrique sur $A$. Soit $\alpha_{v}$ un morphisme de Frobenius sur $A$ pour la place finie $v$. Alors, $\alpha_{v}$ est une isogénie admissible pour $\mathcal{L}$ de poids $\mathrm{q}(\alpha)$.

Démonstration. C'est la proposition 3.3 de [4].

\section{Données}

\subsection{Situation}

Définition 10. On dit qu'une sous-variété $X$ de $\mathbb{P}_{n}$ est projectivement normale si son anneau de coordonnées $S(X)$ est un anneau normal (i.e., intégralement clos).

On peut montrer (cf. par exemple Birkenhake-Lange [2, pp. 190-193]) que $X \subset \mathbb{P}_{n}$ est projectivement normale si et seulement si elle est normale, et pour tout $d \geq 0$ la flèche naturelle

$$
H^{0}\left(\mathbb{P}_{n}, \mathcal{O}_{\mathbb{P}_{n}}(d)\right) \rightarrow H^{0}\left(X, \mathcal{O}_{X}(d)\right)
$$

est surjective.

Concernant les variétés abéliennes plongées de manière projectivement normale, on a le résultat suivant, que l'on trouve dans [2, Theorem 3.1, p. 190].

Proposition 3. Soient $A / k$ une variété abélienne et $\mathcal{L}$ un fibré en droites ample sur $A$. Pour tout $n \geq 3$, le fibré $\mathcal{L}^{\otimes n}$ définit un plongement projectivement normal de $A$ dans un espace projectif $\mathbb{P}_{n}$.

Soit $A / k$ une variété abélienne sur un corps de nombres, munie d'un fibré symétrique ample $\mathcal{L}$. Quitte à travailler avec $\mathcal{L}^{\otimes 4}$ plutôt qu'avec $\mathcal{L}$, on peut supposer que $\mathcal{L}$ est très ample, totalement symétrique et définit un plongement projectivement normal de $A$ dans un projectif $\mathbb{P}_{n}$. On note $\mathcal{M}=\mathcal{L} \otimes \mathcal{L}$ le fibré sur $A \times A$ associé à $\mathcal{L}$. Soit $V$ une $k$-hypersurface irréductible de $A$. On note $I_{V}$ l'idéal de définition de $V$ dans $\mathbb{P}_{n}$. Si $N$ est 
un entier, on a

$$
\begin{aligned}
V \subset A \stackrel{i}{\hookrightarrow} A \times A \hookrightarrow \mathbb{P}_{n} \times \mathbb{P}_{n} \underset{\text { Segre }}{\hookrightarrow} \mathbb{P}_{(n+1)^{2}-1}, \\
x \mapsto(x,[N] x) .
\end{aligned}
$$

Soient $L, T$ deux entiers. On note $\left\{s_{0}, \ldots, s_{l}\right\}$ une base de $H^{0}(A \times A, \mathcal{M})$. On peut, par projective normalité, choisir une base $\left\{Q_{1}, \ldots, Q_{m}\right\}$ du $k$ vectoriel $H^{0}\left(A \times A, \mathcal{M}^{\otimes L}\right)$ telle que tous les $Q_{i}$ sont homogènes de degré $L$ en les $s_{j}$. De plus, on peut aussi voir les $s_{i}$ comme des $(1,1)$-formes homogènes de $k[\mathbf{X}, \mathbf{Y}]$, où $\mathbf{X}=\left(X_{0}, \ldots, X_{n}\right)$ et $\mathbf{Y}=\left(Y_{0}, \ldots, Y_{n}\right)$. Enfin on note $T_{B}$ l'espace tangent à l'origine de la sous-variété abélienne $B=i(A)$ de $A \times A$ définie par $y=[N] x$.

3.2. Choix des paramètres. Soit $C_{0}$ un réel positif. On note $s$ la dimension du stabilisateur de $V$, et $\delta_{i, j}$ le symbole de Kronecker (valant 1 si $i=j$ et 0 sinon). On pose

$$
\begin{aligned}
N_{1} & =\left[C_{0}^{g+2}\left(\log \operatorname{deg}_{\mathcal{L}}(V)\right)^{1+\delta_{g-s, 1}}\left(\log \log \operatorname{deg}_{\mathcal{L}}(V)\right)^{1-2 \delta_{g-s, 1}}\right], \\
m & =\left[\frac{\log \left(C_{0}^{(g+1) / 2}\left(\operatorname{deg}_{\mathcal{L}}(V)\right)^{1 / 2}\left(\log \operatorname{deg}_{\mathcal{L}}(V)\right)^{1 / 2}\left(\log \log \operatorname{deg}_{\mathcal{L}}(V)\right)^{-1}\right)}{\log 2}\right], \\
N & =2^{m+1}, \\
T & =\left[C_{0}^{g+1} \operatorname{deg}_{\mathcal{L}}(V) \log \operatorname{deg}_{\mathcal{L}}(V)\left(\log \log \operatorname{deg}_{\mathcal{L}}(V)\right)^{-3}\right] \\
L & =\left[C_{0}^{g+1 / 2} \operatorname{deg}_{\mathcal{L}}(V) \log \operatorname{deg}_{\mathcal{L}}(V)\left(\log \log \operatorname{deg}_{\mathcal{L}}(V)\right)^{-2}\right] \\
T_{1} & =\left[C_{0}^{g} \operatorname{deg}_{\mathcal{L}}(V)\left(\log \log \operatorname{deg}_{\mathcal{L}}(V)\right)^{-2}\right]
\end{aligned}
$$

Ces paramètres sont choisis de sorte que :

(1) Le nombre $N$ est une puissance de 2 et vérifie l'encadrement

$$
\frac{N}{2} \leq C_{0}^{(g+1) / 2}\left(\operatorname{deg}_{\mathcal{L}}(V)\right)^{1 / 2}\left(\log \operatorname{deg}_{\mathcal{L}}(V)\right)^{1 / 2}\left(\log \log \operatorname{deg}_{\mathcal{L}}(V)\right)^{-1}<N
$$

(2) $N^{2}>L+1$, afin qu'une forme $F$ bihomogène de bidegré $(L, L)$ qui est non-identiquement nulle sur $A \times A$, ne soit pas identiquement nulle sur la sous-variété abélienne $B$.

(3) Le minimum essentiel des variétés intervenant est borné, autrement dit,

$$
N^{2} N_{1} \widehat{\mu}^{\mathrm{ess}}(V) \leq c .
$$

(4) $T>L$, où $T$ va être l'ordre d'annulation dans le lemme de Siegel, et $L$ le degré du polynôme construit.

(5) $T>T_{1}$, puisqu'on ne peut pas par extrapolation espérer un ordre d'annulation meilleur que celui dont on est parti ( $T_{1}$ étant l'ordre d'annulation sur les sous-variétés sur lesquelles on extrapole). 
On fixe un premier $p_{0}$ (ne dépendant que de $A$ ) tel que pour tout premier $p \geq p_{0}$ et pour toute place $v$ divisant $p$, le morphisme de Frobenius $\alpha_{v}$ sur $A$ existe. On fixe alors pour chaque premier $p \geq p_{0}$ une place $v$ au dessus de $p$. On note $\mathcal{P}_{k}$ l'ensemble des places ainsi obtenues.

Dans toute la suite, les inégalités que l'on écrira seront vraies pour tout $\operatorname{deg}_{\mathcal{L}}(V)$ et $C_{0}$ assez grands (i.e., plus grands qu'une constante ne dépendant que du couple $(A, \mathcal{L}))$.

\section{Lemme de Siegel}

$B u t$ : fabriquer un polynôme, $F=\sum_{i=1}^{m} b_{i} Q_{i}$, à coefficients entiers relatifs, en les fonctions abéliennes de $A \times A$, tel que $F$ est de "petite" hauteur, et tel que $F$ s'annule à un ordre supérieur à $T$ sur $i(V)$, le long de $T_{B}$.

En notant $\Theta$ l'application thêta définie sur $T_{A(\mathbb{C})}$ par la composition

$$
T_{A(\mathbb{C})} \stackrel{\exp _{A(\mathbb{C})}}{\longrightarrow} A(\mathbb{C}) \stackrel{\varphi_{\mathcal{L}}}{\longrightarrow} \mathbb{P}_{n}
$$

associée à $\mathcal{L}$, ceci correspond à trouver une solution de petite hauteur au système d'inconnues les $b_{i}$ suivant :

$$
\left.\frac{\partial^{\kappa} F(\Theta(\mathbf{u}+\mathbf{z}), \Theta(N(\mathbf{u}+\mathbf{z})))}{\partial \mathbf{z}^{\kappa}}\right|_{\mathbf{z}=0}=0,
$$

pour tout $|\kappa| \leq T$ et $\mathbf{u} \in T_{A(\mathbb{C})}$ tels que $\Theta(\mathbf{u}) \in V(\bar{k})$.

Lemme 4. Soit $\theta>\widehat{\mu}_{\mathcal{L}}^{\mathrm{ess}}(V)$. Il existe un entier $d_{0}$ tel que si $F$ est une solution du système

$$
\left.\frac{\partial^{\kappa} F(\Theta(\mathbf{u}+\mathbf{z}), \Theta(N(\mathbf{u}+\mathbf{z})))}{\partial \mathbf{z}^{\kappa}}\right|_{\mathbf{z}=0}=0,
$$

pour tout $|\kappa| \leq T$ et $\mathbf{u} \in T_{A(\mathbb{C})}$ tels que $\Theta(\mathbf{u})$ appartient à l'ensemble fini

$$
S_{d_{0}}(\theta)=\left\{x \in V(\bar{k}) \mid \widehat{h}_{\mathcal{L}}(x) \leq \theta,[k(x): k] \leq d_{0}\right\},
$$

alors $F$ est une solution du système (1).

Démonstration. Soit $d \geq 0$ un entier. On peut noter que l'ensemble $S_{d}(\theta)$ est stable sous l'action de $\operatorname{Gal}(\bar{k} / k)$ car $V$ est une $k$-variété. Par ailleurs, on a clairement $S_{d}(\theta) \subset S_{d+1}(\theta)$ pour tout $d \geq 0$. Notons $k[\mathbf{X}]_{L}$ le $k$-espace vectoriel des polynômes homogènes de degré $L$, et $\mathcal{A}_{d}(\theta)$ le sous- $k$-espace vectoriel associé à $S_{d}(\theta)$. La suite $\left(\mathcal{A}_{d}(\theta)\right)_{d \in \mathbb{N}}$ est une suite décroissante d'espaces vectoriels de dimension finie, elle est donc stationnaire. Notons $d_{0}$ l'indice à partir duquel cette suite est stationnaire. Par ailleurs, tous ces espaces contiennent le $k$-vectoriel $\left.I_{V}^{(T)}\right|_{L}$ où $I^{(T)}$ est la puissance symbolique $T$-ième de $I$. Par définition de $d_{0}$, si $P$ est un polynôme homogène de degré $L$ nul sur $S_{d_{0}}$, il appartient à $\mathcal{A}_{d_{0}}(\theta)$, et donc il s'annule sur $\bigcup_{d \geq 0} S_{d}(\theta)$. De plus, par définition du minimum essentiel, $\bigcup_{d \geq 0} S_{d}(\theta)$ est Zariski-dense dans $V$, donc le polynôme $P$ s'annule sur $V$. Ainsi, dans le système (1), on 
peut se restreindre aux $\mathbf{u} \in T_{A(\mathbb{C})}$ tels que $\Theta(\mathbf{u})$ appartient à $S_{d_{0}}(\theta)$. Par un théorème classique de Northcott, cet ensemble est fini.

On appelle (2) le nouveau système ainsi obtenu. On passe maintenant à une estimation du rang.

Lemme 5. Il existe une constante $c_{1}$ telle que le rang du système (1) est majoré par $c_{1} T\left(L N^{2}\right)^{g-1} \operatorname{deg}_{\mathcal{L}}(V)$.

Démonstration. Il s'agit du lemme (ou plutôt de la preuve du lemme) 5.1 de [4]. En effet, dans ce lemme, les auteurs de [4] cherchent à obtenir une majoration du rang du système

$$
\left.\frac{\partial^{\kappa} F(\Theta(\mathbf{u}+\mathbf{z}), \Theta(N(\mathbf{u}+\mathbf{z})))}{\partial \mathbf{z}^{\kappa}}\right|_{\mathbf{z}=0}=0,
$$

où $\mathbf{u}$ est le logarithme d'un point $Q$ fixé. L'idée est d'appliquer "l'astuce de Philippon-Waldschmidt" (voir [13, lemme 6.7]). Pour majorer le rang de ce système, ils se donnent une variété $V$ de dimension $d$ contenant le point $Q$, et ils majorent le système

$$
\left.\frac{\partial^{\kappa} F(\Theta(\mathbf{u}+\mathbf{z}), \Theta(N(\mathbf{u}+\mathbf{z})))}{\partial \mathbf{z}^{\kappa}}\right|_{\mathbf{z}=0}=0,
$$

pour tout $|\kappa| \leq T$ et $\mathbf{u} \in T_{A(\mathbb{C})}$ tels que $\Theta(\mathbf{u}) \in V(\bar{k})$. Ils obtiennent comme majorant du rang de ce système le nombre $c_{1} T^{g-d}\left(L N^{2}\right)^{d} \operatorname{deg}_{\mathcal{L}}(V)$. (On remplace dans leurs notations $T_{0}$ par $T$.) En appliquant ceci à l'hypersurface $V$ considérée, on obtient donc le résultat cherché.

On peut maintenant énoncer le lemme de Siegel qui nous intéresse. Si $F=\sum a_{i} \mathbf{X}^{i}$ est un polynôme à coefficients dans $\bar{k}$, on définit classiquement sa hauteur $h(F)$ comme étant la hauteur logarithmique absolue du point projectif défini par 1 et tous les coefficients $a_{i}$ de $F$.

L'objectif de l'article consiste à montrer que

$$
\operatorname{deg}_{\mathcal{L}}(V) \widehat{\mu}_{\mathcal{L}}^{\operatorname{ess}}(V)>\frac{c(A / k, \mathcal{L})}{\log \operatorname{deg}_{\mathcal{L}}(V)^{\alpha}} .
$$

On peut donc toujours supposer que $\widehat{\mu}_{\mathcal{L}}^{\text {ess }}(V)$ est strictement inférieur à 1.

Proposition 4. Il existe une solution $F=\sum_{i=1}^{m} b_{i} Q_{i}, b_{i} \in \mathbb{Z}$, du système (1) de degré $L$ et de hauteur

$$
h(F) \leq c_{2} C_{0}^{(g+1) / 2} \operatorname{deg}_{\mathcal{L}}(V) \log \operatorname{deg}_{\mathcal{L}}(V)\left(\log \log \operatorname{deg}_{\mathcal{L}}(V)\right)^{-2} .
$$

Démonstration. Soit $1>\theta>\widehat{\mu}_{\mathcal{L}}^{\text {ess }}(V)$. Par le lemme 4 il suffit, pour trouver une solution du (1), de trouver une solution du (2). Ceci remarqué, on est ramené à une preuve classique. On suit pour cela la preuve du lemme 5.4 de $[4]$. 
On commence par évaluer la hauteur du (2). Le système (18) ainsi que l'inégalité qui suit à la page 42 de [4] nous indiquent que la hauteur de chaque coefficient du système est majorée par

$$
c_{4}^{\prime} L N^{2} \theta+T(\log (T+L)+\log N) .
$$

Par ailleurs, le nombre d'inconnues $I$ est $\operatorname{dim} H^{0}\left(A \times A, \mathcal{M}^{\otimes L}\right)$. Le théorème de Riemann-Roch pour les variétés abéliennes nous assure que $I$ vérifie l'encadrement

$$
c_{5} L^{2 g} \leq I \leq c_{6} L^{2 g} .
$$

Notons $M$ la matrice du système (2). Elle est définie sur $k$, donc si $\mathfrak{B}$ dénote le noyau de $M$, il est muni d'une $k$-structure. Si $\mathfrak{b}$ est la dimension de $\mathfrak{B}$, on a $\mathfrak{b}=I-\operatorname{rg}(M)$. Le lemme de Siegel classique (cf. par exemple Schmidt [15, Lemma IV.B, p. 10]) nous indique alors qu'il existe une solution non triviale comme recherchée, de hauteur

$$
h(F) \leq c_{7} \frac{h(\mathfrak{B})}{\mathfrak{b}}
$$

où $h(\mathfrak{B})$ représente la hauteur du point $\mathfrak{B}$ défini dans la grassmannienne correspondante. De plus, le lemme IV, p. 10, de [15] nous indique que $h(\mathfrak{B})=$ $h\left(\mathfrak{B}^{\perp}\right)$. L'espace $\mathfrak{B}^{\perp}$ étant l'espace vectoriel engendré par les colonnes de $M$, sa hauteur est par définition majorée par celle d'un mineur maximal $\Delta_{\max }$ de $M$. Cette dernière hauteur est majorée par

$$
\begin{aligned}
h\left(\Delta_{\max }\right) & \leq c_{8} \operatorname{rg}(M)\left(\log (\operatorname{rg} M)+c_{4}^{\prime} L N^{2} \theta+T(\log (T+L)+\log N)\right) \\
& \leq c_{9} T\left(L N^{2}\right)^{g-1} \operatorname{deg}_{\mathcal{L}}(V)\left(\log \operatorname{deg}_{\mathcal{L}}(V)+2 T \log T\right),
\end{aligned}
$$

la première inégalité découlant de (5), et la seconde du lemme 5 en utilisant également le fait que $T>L$ et $T>N$. En remplacant $T$ et $N$ par leur valeur, on obtient

$$
\begin{aligned}
h\left(\Delta_{\max }\right) \leq & c_{10} L^{g-1} C_{0}^{(g+1)^{2}} \\
& \times\left(\operatorname{deg}_{\mathcal{L}}(V) \log \operatorname{deg}_{\mathcal{L}}(V)\right)^{g+2}\left(\log \log \operatorname{deg}_{\mathcal{L}}(V)\right)^{-2(g+2)} .
\end{aligned}
$$

De plus, par l'inégalité (6), et par le choix de $L$, on a

$$
\mathfrak{b} \geq c_{11} L^{g-1}\left(L^{g+1}-c_{1} T\left(N^{2}\right)^{g-1} \operatorname{deg}_{\mathcal{L}}(V)\right) \geq c_{12} L^{g+1} L^{g-1} .
$$

En remplaçant $L^{g+1}$ par sa valeur, on obtient la minoration

$$
\begin{aligned}
\mathfrak{b} \geq & c_{13} C_{0}^{(g+1)(g+1 / 2)} L^{g-1} \\
& \times\left(\operatorname{deg}_{\mathcal{L}}(V) \log \operatorname{deg}_{\mathcal{L}}(V)\right)^{g+1}\left(\log \log \operatorname{deg}_{\mathcal{L}}(V)\right)^{-2(g+1)} .
\end{aligned}
$$

On reprend maintenant l'inégalité (7) en remplaçant les paramètres par leurs valeurs. On obtient ainsi l'inégalité

$$
h(F) \leq c_{2} C_{0}^{(g+1) / 2} \operatorname{deg}_{\mathcal{L}}(V) \log \operatorname{deg}_{\mathcal{L}}(V)\left(\log \log \operatorname{deg}_{\mathcal{L}}(V)\right)^{-2} .
$$


Remarque 4. La fonction auxiliaire $F$ ainsi construite est une forme bihomogène de bidegré $(L, L)$ non identiquement nulle sur $A \times A$. Elle n'est donc pas identiquement nulle sur $B$ car $N^{2}>L+1$.

5. Extrapolation. On veut montrer dans ce paragraphe que $F$ s'annule sur $i\left(\alpha_{v}(V)\right)$ pour $v \in \mathcal{P}_{k}$ appartenant à un ensemble convenable. Pour cela, on utilise un argument remontant à Dobrowolski [6] dans son célèbre article sur la conjecture de Lehmer sur les points pour $\mathbb{G}_{m}$. Cet argument a été réécrit et adapté dans le cadre des variétés abéliennes de type C.M. dans l'article [4] suivant des idées de Laurent [9]. Ce que l'on fait ici repose sur le paragraphe 6 de [4].

Proposition 5. La fonction auxiliaire $F$ est nulle sur $i\left(\alpha_{v}(V)\right)$ à un ordre supérieur à $T_{1}$ le long de $T_{B}$ pour toute place $v \in \mathcal{P}_{k}$ de norme comprise entre $\frac{1}{2} N_{1}$ et $N_{1}$.

Démonstration. Soit $1>\theta>\widehat{\mu}_{\mathcal{L}}^{\text {ess }}(V)$. Il s'agit de reprendre la proposition 6.5 de [4]. On conserve donc leurs notations. Soient $v$ une place comme dans l'énoncé, $R$ un point de $V(\bar{k})$ défini sur une extension $k^{\prime}$ de $k$ de hauteur normalisée inférieure à $\theta$, et $w$ une place de $k^{\prime}$ au-dessus de $v$. Notons $\mathbf{R}=\left(R_{0}, \ldots, R_{n}\right)$ un système de coordonnées projectives de $R$ dans $\mathcal{O}_{w}$, telles que $\|\mathbf{R}\|_{w}=1$. Soit $\partial^{\kappa}$ un opérateur différentiel d'ordre $|\kappa| \leq T_{1}$ le long de $T_{B(\mathbb{C})}$. L'application du petit théorème de Fermat dans le cadre des variétés abéliennes nous donne

$$
\left|\partial^{\kappa} F\left(\mathbf{F}_{\alpha_{v}}(\mathbf{R}), \mathbf{F}^{(N)} \circ \mathbf{F}_{\alpha_{v}}(\mathbf{R})\right)\right|_{w} \leq\left|\pi_{v}\right|_{w}^{T-|\kappa|},
$$

où $\mathbf{F}_{\alpha_{v}}$ et $\mathbf{F}^{(N)}$ sont des formes homogènes de $\mathcal{O}_{k}[\mathbf{X}]$ de degré respectif $\mathrm{N}(v)$ et $4^{m+1}$, représentant respectivement l'endomorphisme de Frobenius sur $A$ associé à $v$, et la multiplication par $N=2^{m+1}$. Il s'agit de l'inégalité (20), p. 47 , de [4].

On veut maintenant sommer sur toutes les places $w$ au-dessus de $v$. Malheureusement, le choix du système de coordonnées projectives pour $R$ dépend de $w$. On est donc obligé d'alourdir les notations pour pallier ce problème. Soient $S, S_{N}, S_{\alpha_{v}}, S_{N, \alpha_{v}}$ des coordonnées projectives non nulles de $R, \mathbf{F}^{(N)}(\mathbf{R}), \mathbf{F}_{\alpha_{v}}(\mathbf{R}), \mathbf{F}^{(N)} \circ \mathbf{F}_{\alpha_{v}}(\mathbf{R})$ respectivement. On note de plus $S_{w, N}, S_{w, \alpha_{v}}, S_{w, N, \alpha_{v}}$ des coordonnées de ces points de valeur absolue $w$ adique maximale.

Soit maintenant $\partial^{\kappa}$ un opérateur différentiel de longueur minimale pour lequel $\partial^{\kappa} F\left(\mathbf{F}_{\alpha_{v}}(\mathbf{R}), \mathbf{F}^{(N)} \circ \mathbf{F}_{\alpha_{v}}(\mathbf{R})\right)$ est non nul. Si $|\kappa|$ est supérieur à $T_{1}$, on a gagné. Sinon on applique la formule de Leibniz en utilisant que $F$ est bihomogène de bidegré $(L, L)$. On a donc

$$
\partial^{\kappa} F\left(\frac{\mathbf{F}_{\alpha_{v}}(\mathbf{R})}{S_{\alpha_{v}}}, \frac{\mathbf{F}^{(N)}\left(\mathbf{F}_{\alpha_{v}}(\mathbf{R})\right)}{S_{N, \alpha_{v}}}\right)=\frac{\partial^{\kappa} F\left(\mathbf{F}_{\alpha_{v}}(\mathbf{R}), \mathbf{F}^{(N)}\left(\mathbf{F}_{\alpha_{v}}(\mathbf{R})\right)\right)}{S_{\alpha_{v}}^{L} S_{N, \alpha_{v}}^{L}} .
$$


Or ceci est égal à

$$
\partial^{\kappa} F\left(\frac{\mathbf{F}_{\alpha_{v}}(\mathbf{R})}{S_{w, \alpha_{v}}}, \frac{\mathbf{F}^{(N)}\left(\mathbf{F}_{\alpha_{v}}(\mathbf{R})\right)}{S_{w, N, \alpha_{v}}}\right) \cdot \frac{\left(S_{w, \alpha_{v}} S_{w, N, \alpha_{v}}\right)^{L}}{\left(S_{\alpha_{v}} S_{N, \alpha_{v}}\right)^{L}} .
$$

On réécrit alors l'inégalité (10) en passant au log, en sommant sur toutes les places $w$ au-dessus de $v$ et en notant $n_{w}$ les degrés locaux :

$$
\begin{aligned}
& \sum_{w / v} n_{w} \log \left(\left|\partial^{\kappa} F\left(\frac{\mathbf{F}_{\alpha_{v}}(\mathbf{R})}{S_{\alpha_{v}}}, \frac{\mathbf{F}^{(N)}\left(\mathbf{F}_{\alpha_{v}}(\mathbf{R})\right)}{S_{N, \alpha_{v}}}\right)\right|_{w}\right) \\
& \quad \leq(T-|\kappa|) \sum_{w / v} n_{w} \log \left(\left|\pi_{v}\right|_{w}\right)+L \sum_{w / v} n_{w} \log \left(\frac{\left|S_{w, \alpha_{v}} S_{w, N, \alpha_{v}}\right|_{w}}{\left|S_{\alpha_{v}} S_{N, \alpha_{v}}\right|_{w}}\right) .
\end{aligned}
$$

Or

$$
\sum_{w / v} n_{w} \log \left(\left|\pi_{v}\right|_{w}\right)=\left[k^{\prime}: k\right] \log \left(\left|\pi_{v}\right|_{v}\right) \leq-\left[k^{\prime}: k\right] \log (\mathrm{N}(v)) .
$$

De plus, on peut voir que

$$
\begin{aligned}
\sum_{w / v} n_{w} \log \left(\frac{\left|S_{w, \alpha_{v}} S_{w, N, \alpha_{v}}\right|_{w}}{\left|S_{\alpha_{v}} S_{N, \alpha_{v}}\right| w}\right) \\
\leq\left[k^{\prime}: k\right]\left(h_{\mathcal{L}}\left(\alpha_{v}(R)\right)+h_{\mathcal{L}}\left(N \alpha_{v}(R)\right)\right) \\
\leq\left[k^{\prime}: k\right]\left(\mathrm{N}(v) \widehat{h}_{\mathcal{L}}(R)+N^{2} \mathrm{~N}(v) \widehat{h}_{\mathcal{L}}(R)+c_{14}\right) .
\end{aligned}
$$

C'est l'inégalité (21), p. 49, de [4]. En tenant compte du fait que le point $R$ est supposé de hauteur (de Néron-Tate) inférieure à $\theta$, et en injectant ceci dans (11), on obtient, en remplaçant les paramètres par leur valeur, l'inégalité

$$
\begin{aligned}
-\frac{1}{\left[k^{\prime}: k\right]} \sum_{w / v} n_{w} \log \left(\left|\partial^{\kappa} F\left(\frac{\mathbf{F}_{\alpha_{v}}(\mathbf{R})}{S_{\alpha_{v}}}, \frac{\mathbf{F}^{(N)}\left(\mathbf{F}_{\alpha_{v}}(\mathbf{R})\right)}{S_{N, \alpha_{v}}}\right)\right|_{w}\right) \\
\geq \frac{1}{2} T \log N_{1} .
\end{aligned}
$$

Il reste à majorer le membre de gauche de cette dernière inégalité. Notons $A$ ce membre de gauche. Par définition de la hauteur (absolue logarithmique) projective, on a

$$
\begin{aligned}
A & \leq h\left(\left(\partial^{\kappa} F\left(\frac{\mathbf{F}_{\alpha_{v}}(\mathbf{R})}{S_{\alpha_{v}}}, \frac{\mathbf{F}^{(N)}\left(\mathbf{F}_{\alpha_{v}}(\mathbf{R})\right)}{S_{N, \alpha_{v}}}\right)\right)^{-1}\right) \\
& =h\left(\partial^{\kappa} F\left(\frac{\mathbf{F}_{\alpha_{v}}(\mathbf{R})}{S_{\alpha_{v}}}, \frac{\mathbf{F}^{(N)}\left(\mathbf{F}_{\alpha_{v}}(\mathbf{R})\right)}{S_{N, \alpha_{v}}}\right)\right),
\end{aligned}
$$

ceci ayant un sens grace à l'hypothèse de non nullité de $\partial^{\kappa} F(\ldots)$. Il ne reste maintenant plus qu'à majorer cette dernière hauteur. Il s'agit d'un calcul 
classique (cf. par exemple [4, p. 50]). On obtient

$$
A \leq c_{15}\left(T_{1} \log \left(T_{1}+L\right)+L N^{2} \mathrm{~N}(v) \theta+h(F)\right) .
$$

Finalement, en mettant ensemble les inégalités (14) et (15), on obtient

$$
T \log N_{1} \leq c_{16} T_{1} \log \left(T_{1}+L\right)+c_{16} L N^{2} N_{1} \theta+c_{16} h(F) .
$$

On remplace les différents paramètres par leurs valeurs, et on obtient pour le membre de gauche de l'inégalité

$$
C_{0}^{g+1} \operatorname{deg}_{\mathcal{L}}(V) \log \operatorname{deg}_{\mathcal{L}}(V)\left(\log \log \operatorname{deg}_{\mathcal{L}}(V)\right)^{-2},
$$

et pour le membre de droite

$$
c_{17} C_{0}^{g} \operatorname{deg}_{\mathcal{L}}(V) \log \operatorname{deg}_{\mathcal{L}}(V)\left(\log \log \operatorname{deg}_{\mathcal{L}}(V)\right)^{-2} .
$$

Dès que $C_{0}$ est assez grand, on aboutit à une contradiction.

6. Conclusion. On commence par minorer le degré de l'union des variétés transformées de $V$.

Proposition 6. Soient $A$ une variété abélienne sur $k$ de dimension $g \geq 1, \mathcal{L}$ un fibré en droites ample sur $A$, et $V$ une sous- $k$-variété stricte de $A$, irréductible sur $k$. On suppose que $V$ n'est pas une réunion de sousvariétés de torsion de $A$, et que le nombre $M$ de composantes géométriques de $V$ est majoré par $c_{3} \operatorname{deg}_{\mathcal{L}}(V)^{g}$. On considère enfin un ensemble d'isogénies $\beta_{v}$ admissibles, deux à deux premières entre elles, avec $v \in \mathcal{P}_{k}^{1}=\mathcal{P}_{k} \cap$ $\llbracket N_{1} / 2, N_{1} \rrbracket$. On $a$

$$
\operatorname{deg}_{\mathcal{L}}\left(\bigcup_{v \in \mathcal{P}_{k}^{1}} \beta_{v}(V)\right) \geq c_{4} \frac{\operatorname{deg}_{\mathcal{L}}(V) N_{1}^{g-\operatorname{dim} G_{V}}}{\log N_{1}} .
$$

Démonstration. Soit $W$ une composante géométriquement irréductible de $V$. Pour $v \in \mathcal{P}_{k}^{1}$, on a, $\beta_{v}$ étant définie sur $k$,

$$
\operatorname{card}\left(\operatorname{ker}\left(\beta_{v}\right) \cap G_{\sigma(W)}\right)=\operatorname{card}\left(\operatorname{ker}\left(\beta_{v}\right) \cap G_{W}\right) .
$$

Par ailleurs, comme $W$ n'est pas une sous-variété de torsion de $A$ (sinon $V$ serait réunion de telles sous-variétés), le point (2) du lemme 3 nous indique que l'égalité $\beta_{v}(W)=\beta_{v}(\sigma(W)$ ) (et $W \neq \sigma(W)$ ) n'est possible que pour au plus $(\log M) / 2 \leq c_{17} \log \operatorname{deg}_{\mathcal{L}}(V)$ éléments $v$ de $\mathcal{P}_{k}^{1}$. Notons $\mathcal{P}_{k}^{1 \star}$ le sousensemble de $\mathcal{P}_{k}^{1}$ obtenu en enlevant ces éléments. Le théorème de Chebotarev nous indique que

$$
\operatorname{card}\left(\mathcal{P}_{k}^{1}\right) \geq c_{18} \frac{N_{1}}{\log N_{1}} .
$$

En remplaçant $N_{1}$ par sa valeur, on constate que

$$
\operatorname{card}\left(\mathcal{P}_{k}^{1 \star}\right) \geq \frac{1}{2} \operatorname{card}\left(\mathcal{P}_{k}^{1}\right) .
$$


En utilisant l'additivité du degré et les lemmes précédents, on a

$$
\operatorname{deg}_{\mathcal{L}}\left(\bigcup_{v \in \mathcal{P}_{k}^{1}} \beta_{v}(V)\right) \geq \operatorname{deg}_{\mathcal{L}}\left(\bigcup_{v \in \mathcal{P}_{k}^{1 \star}, \sigma \in \operatorname{Gal}(\bar{k} / k)} \beta_{v}(\sigma(W))\right) .
$$

Par le lemme 3, ceci est supérieur à

$$
\sum_{v \in \mathcal{P}_{k}^{1 \star}} \operatorname{deg}_{\mathcal{L}}\left(\bigcup_{\sigma \in \operatorname{Gal}(\bar{k} / k)} \beta_{v}(\sigma(W))\right) .
$$

Enfin, le lemme 1 nous donne l'inégalité

$$
\operatorname{deg}_{\mathcal{L}}\left(\bigcup_{v \in \mathcal{P}_{k}^{1}} \beta_{v}(V)\right) \geq M \operatorname{deg}_{\mathcal{L}}(W) \sum_{v \in \mathcal{P}_{k}^{1 \star}} \frac{\mathrm{q}\left(\beta_{v}\right)^{\operatorname{dim} V}}{\left|G_{W} \cap \operatorname{ker}\left(\beta_{v}\right)\right|} .
$$

Le lemme 2.1(ii) de [4] nous indique que

$$
\operatorname{deg}_{\mathcal{L}}\left(G_{W}\right)=\left[G_{W}: G_{W}^{0}\right] \operatorname{deg}_{\mathcal{L}}\left(G_{W}^{0}\right) \leq \operatorname{deg}_{\mathcal{L}}(V)^{g} .
$$

En particulier on en déduit que

$$
\left[G_{W}: G_{W}^{0}\right] \leq \operatorname{deg}_{\mathcal{L}}(V)^{g} .
$$

De plus, les $\beta_{v}$ étant premiers entre eux, on a

$$
\prod_{v \in \mathcal{P}_{k}^{1 \star}}\left|\operatorname{ker}\left(\beta_{v}\right) \cap G_{W}\right|=\left|\operatorname{ker}\left(\prod_{v \in \mathcal{P}_{k}^{1 \star}} \beta_{v}\right) \cap G_{W}\right| .
$$

En appliquant le lemme 2, on en déduit

$$
\left|\operatorname{ker}\left(\prod_{v \in \mathcal{P}_{k}^{1 \star}} \beta_{v}\right) \cap G_{W}\right| \leq\left[G_{W}: G_{W}^{0}\right]\left(\prod_{v \in \mathcal{P}_{k}^{1 \star}} \mathrm{q}\left(\beta_{v}\right)\right)^{s} .
$$

En appliquant l'inégalité arithmético-géométrique, on obtient

(21) $\operatorname{deg}_{\mathcal{L}}\left(\bigcup_{v \in \mathcal{P}_{k}^{1}} \beta_{v}(V)\right) \geq c_{4} \operatorname{deg}_{\mathcal{L}}(V) \frac{\operatorname{card}\left(\mathcal{P}_{k}^{1 \star}\right) N_{1}^{g-1}}{\left(\prod_{v \in \mathcal{P}_{k}^{1 \star}}\left|\operatorname{ker}\left(\beta_{v}\right) \cap G_{W}\right|\right)^{1 / \operatorname{card}\left(\mathcal{P}_{k}^{1 \star}\right)}}$

En appliquant l'inégalité (20) et la minoration du cardinal de $\mathcal{P}_{k}^{1 \star}$, on obtient

$$
\begin{aligned}
\operatorname{deg}_{\mathcal{L}}\left(\bigcup_{v \in \mathcal{P}_{k}^{1}} \beta_{v}(V)\right) \\
\quad \geq c_{4} \frac{\operatorname{deg}_{\mathcal{L}}(V) N_{1}^{g}}{\log N_{1}\left[G_{W}: G_{W}^{0}\right]^{\left(\log N_{1}\right) /\left(c_{56} N_{1}\right)} \prod_{v \in \mathcal{P}_{k}^{1 \star}} \mathrm{q}\left(\beta_{v}\right)^{g / \mathcal{P}_{k}^{1 \star}}} .
\end{aligned}
$$

Enfin par définition de $\mathcal{P}_{k}^{1}$, on a la majoration $\mathrm{q}\left(\beta_{v}\right) \leq N_{1}$. En appliquant ceci et la majoration de l'indice de $G_{W}^{0}$ dans $G_{W}$, on a

$$
\operatorname{deg}_{\mathcal{L}}\left(\bigcup_{v \in \mathcal{P}_{k}^{1}} \beta_{v}(V)\right) \geq c_{4} \frac{\operatorname{deg}_{\mathcal{L}}(V) N_{1}^{g-\operatorname{dim} G_{V}}}{\log N_{1}} .
$$


Remarque 5. C'est uniquement pour assurer l'inégalité (18) que l'on est conduit à choisir l'exposant du terme $\log \log$ dans $N_{1}$ tel qu'indiqué, plutôt que l'exposant $-1 /\left(g-\operatorname{dim} G_{V}\right)$ qui serait plus proche des choix de [1]. Cette amélioration dans [1] est rendue possible par la résolution de deux complications techniques : passage à une hypersurface secondaire explicitement construite, et raffinement galoisien.

Ceci étant, on peut maintenant démontrer le théorème recherché.

Démonstration. On suppose par l'absurde que l'inégalité du théorème à prouver n'est pas vérifiée pour $C_{0}=c(A / k, \mathcal{L})^{-1 /(g+3)}$ assez grand (i.e. $c(A / k, \mathcal{L})$ suffisamment petit). Dans cette preuve, on considère, pour alléger les notations, la variété abélienne $A$ comme étant plongée dans $\mathbb{P}_{n}$. Notons $\mathcal{Z}$ l'hypersurface sur $k$ de $\mathbb{P}_{n}$ associée à la forme $F \circ \varphi$ de degré $\left(N^{2}+1\right) L$. Par choix de $N$ (à savoir $\left(N^{2}+1\right)>L$ ), la variété $\mathcal{Z} \cap A$ est une hypersurface de $A$. De plus, par la proposition 5 , on sait que cette hypersurface contient les variétés irréductibles $\alpha_{v}(V)$ avec une multiplicité supérieure à $T_{1}$, pour toute place $v$ de norme comprise entre $\frac{1}{2} N_{1}$ et $N_{1}$. Donc le théorème de Bézout géométrique nous donne

$$
T \operatorname{deg}_{\mathcal{L}}(V)+T_{1} \operatorname{deg}_{\mathcal{L}}\left(\bigcup_{N_{1} / 2 \leq \mathrm{N}(v) \leq N_{1}} \alpha_{v}(V)\right) \leq \operatorname{deg}_{\mathcal{L}}(A) L\left(N^{2}+1\right) .
$$

Cette inégalité implique en particulier que le nombre $M$ de composantes géométriquement irréductibles de $V$ est majoré par une expression de la forme $c_{3} \operatorname{deg}_{\mathcal{L}}(V)^{g}$. On peut donc appliquer la proposition 6 avec $\beta_{v}=\alpha_{v}$. Celle-ci et l'inégalité obtenue par le théorème de Bézout nous fournissent l'inégalité

$$
T_{1} \frac{\operatorname{deg}_{\mathcal{L}}(V) N_{1}^{g-\operatorname{dim} G_{V}}}{\log N_{1}} \leq c_{19}(A) L\left(N^{2}+1\right) .
$$

On remplace maintenant les paramètres par leurs valeurs pour conclure. Si $C_{0}$ est assez grand, l'inégalité est contredite : si $s=g-1$, les deux membres sont du même ordre de grandeur, or, dans le membre de gauche, on a un terme constant de la forme $C_{0}^{2 g+2}$, alors que dans le membre de droite, le terme constant est de la forme $C_{0}^{2 g+3 / 2}$; sinon l'ordre de grandeur du membre de gauche est supérieur à celui du terme de droite. (En fait, $T_{1}$ est construit exactement pour contredire cette inégalité.)

\section{Références}

[1] F. Amoroso et S. David, Minoration de la hauteur normalisée des hypersurfaces, Acta Arith. 92 (2000), 339-365.

[2] C. Birkenhake and H. Lange, Complex Abelian Varieties, Grundlehren Math. Wiss. 302, Springer, 1992. 
[3] J.-B. Bost, H. Gillet, and C. Soulé, Heights of projective varieties and positive Green forms, J. Amer. Math. Soc. 7 (1994), 903-1022.

[4] S. David et M. Hindry, Minoration de la hauteur de Néron-Tate sur les variétés abéliennes de type C.M., J. Reine Angew. Math. 529 (2000), 1-74.

[5] S. David et P. Philippon, Minorations des hauteurs normalisées des sous-variétés de variétés abéliennes, dans : Number Theory (Tiruchirapalli, 1996), Contemp. Math. 210, Amer. Math. Soc., 1998, 333-364.

[6] E. Dobrowolski, On a question of Lehmer and the number of irreducible factors of a polynomial, Acta Arith. 34 (1979), 391-401.

[7] M. Hindry, Autour d'une conjecture de Serge Lang, Invent. Math. 94 (1988), 575603.

[8] M. Hindry and J. Silverman, Diophantine Geometry. An Introduction, Grad. Texts in Math. 201, Springer, 2000.

[9] M. Laurent, Minoration de la hauteur de Néron-Tate, dans : M.-J. Bertin (ed.), Séminaire de théorie des nombres de Paris (1981-1982), Progr. Math. 38, Birkhäuser, Boston, 1983, 137-151.

[10] P. Philippon, Sur des hauteurs alternatives I, Math. Ann. 289 (1991), 255-283.

[11] —, Sur des hauteurs alternatives II, Ann. Inst. Fourier (Grenoble) 44 (1994), 10431065 .

[12] —, Sur des hauteurs alternatives III, J. Math. Pures Appl. 74 (1995), 345-365.

[13] P. Philippon et M. Waldschmidt, Formes linéaires de logarithmes sur les groupes algébriques commutatifs, Illinois J. Math. 32 (1988), 281-314.

[14] N. Ratazzi, Densité de points et minoration de hauteur, J. Number Theory, to appear; doi:10.1016/j.jnt.2003.12.007.

[15] W. Schmidt, Diophantine Approximations and Diophantine Equations, Lecture Notes in Math. 1467, Springer, 1991.

[16] G. Shimura and Y. Taniyama, Complex multiplication of abelian varieties and its applications to number theory, Publ. Math. Soc. Japan 6, Math. Soc. Japan, 1961.

[17] C. Soulé, Géométrie d'Arakelov et théorie des nombres transcendants, Journées Arithmétiques, 1989 (Luminy, 1989), Astérisque 198-200 (1991), 355-371.

[18] E. Ullmo, Positivité et discrétion des points algébriques des courbes, Ann. of Math. 147 (1998), 167-179.

[19] S. Zhang, Small points and adelic metrics, J. Algebraic Geom. 4 (1995), 281-300.

[20] —, Equidistribution of small points on abelian varieties, Ann. of Math. 147 (1998), 159-165.

Institut de mathématiques

Projet théorie des nombres, UMR 7586

Université Paris 6

Case 247

4 place Jussieu

75252 Paris, France

E-mail: ratazzi@math.jussieu.fr 